Canadian

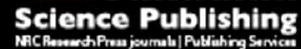

Canadian Journal of Microbiology Revue canadienne de de microbiologie

\title{
Molecular characterization of pathogenic and non- pathogenic fowl aviadenovirus serotype 11 isolates
}

\begin{tabular}{|r|l|}
\hline Journal: & Canadian Journal of Microbiology \\
\hline Manuscript ID & cjm-2016-0297.R1 \\
\hline Manuscript Type: & Article \\
\hline Date Submitted by the Author: & 27-Jun-2016 \\
\hline Complete List of Authors: & $\begin{array}{l}\text { Slaine, P.D.; University of Guelph } \\
\text { Ackford, J.G. ; University of Guelph } \\
\text { Kropinski, Andrew; University of Guelph } \\
\text { Kozak, R.A.; University of Guelph } \\
\text { Krell, Peter; Virology, Genetics and Molecular Biology, } \\
\text { Nagy, Eva; University of Guelph Ontario Veterinary College, Pathobiology }\end{array}$ \\
\hline Keyword: & $\begin{array}{l}\text { aviadenovirus, fowl aviadenovirus serotype 11, genomic sequences, host- } \\
\text { pathogen interaction }\end{array}$ \\
\hline &
\end{tabular}




\section{Molecular characterization of pathogenic and non-pathogenic fowl aviadenovirus serotype 11 isolates}

Patrick D. Slaine*1, James G. Ackford*1 ${ }^{1}$, Andrew M. Kropinski ${ }^{1}$, Robert A. Kozak ${ }^{1}$, Peter J. Krell ${ }^{2}$ and Éva Nagy ${ }^{1}$

${ }^{1}$ Department of Pathobiology, Ontario Veterinary Collage and ${ }^{2}$ Department of Molecular and Cellular Biology, College of Biological Sciences, University of Guelph, 50 Stone Road East, Guelph, ON N1G 2W1, Canada

E-mail addresses: P.D. Slaine: pslaine@mail.uoguelph.ca; J.G. Ackford: jackford@uoguelph.ca; A.M. Kropinski: akropins@uoguelph.ca; R.A. Kozak: rkozak@uoguelph.ca; P.J.Krell: pkrell@uoguelph.ca;

É. Nagy: enagy@uoguelph.ca

Corresponding author: Éva Nagy

* These authors contributed equally

Current E-mail addresses: Patrick.Slaine@Dal.ca; james@froggabio.com; robert.kozak@phacaspc.gc.ca 


\begin{abstract}
Fowl aviadenoviruses, many of which are of importance in veterinary medicine, are classified into five species. In this study, a pathogenic isolate and a non-pathogenic isolate of fowl aviadenovirus serotype 11 (FAdV-11) of species Fowl aviadenovirus D were characterized. Growth rates were analyzed for the two isolates, showing notable differences. The complete genomic sequences of the viruses were fully determined and were analyzed. The genomes of the two isolates showed $98.1 \%$ sequence identity, and revealed six nonsynonymous mutations between the Ontario isolates. Two of the six mutations were also found in the sequences of recently published pathogenic Chinese fowl aviadenovirus 11 isolates, suggesting potential molecular markers that could be associated with pathogenesis. Deletions were found in the L5 region within the overlapping coding sequences for the $100 \mathrm{kDa}, 22 \mathrm{kDa}$, and $33 \mathrm{kDa}$ proteins and these were found in only the non-pathogenic isolates. This molecular pattern was identified in FAdV-9, another non-pathogenic FAdV-D species virus. Furthermore, the tandem repeat regions varied dramatically, the pathogenic isolates contained a reduced number of tandem repeats compared to the non-pathogenic isolates. Lastly, a protein produced early in infection was analyzed using bioinformatics to determine its role in disease. This study highlights several candidate molecular determinants of avian adenovirus genomes related to pathogenicity.
\end{abstract}

Keywords: aviadenovirus, fowl aviadenovirus serotype 11, genomic sequence, host-pathogen interaction 


\section{Introduction}

Adenoviruses (AdVs) can infect a wide range of hosts, however avian adenoviruses are distinct from other AdVs, and infect only avian species. The genus Aviadenovirus contains 12 serotypes of fowl aviadenoviruses (FAdV) that are grouped into five species, Fowl aviadenovirus $A$ through Fowl aviadenovirus E. In poultry, FAdVs are considered ubiquitous, and while many of these viruses are non-pathogenic, some of them are associated with diseases such as inclusion body hepatitis (IBH) caused by FAdV-2, -6, -7, -8, and -11 (Hess 2013). The complete nucleotide sequences are available for at least one member of each species: FAdV-1 in Fowl aviadenovirus A (Chiocca et al. 1996), FAdV-5 in Fowl aviadenovirus B (Marek et al. 2013), FAdV-4 in Fowl aviadenovirus $C$ (Griffin and Nagy 2011), FAdV-9 in Fowl aviadenovirus D (Ojkic and Nagy 2000) and FAdV-8 in Fowl aviadenovirus E (Grgic et al. 2011).

Inclusion body hepatitis is an economically important disease of poultry, infecting birds worldwide including Canada (Ojkic et al. 2008a). The dominant viruses in Canada are of serotype $8(\mathrm{FAdV}-8)$ and serotype $11(\mathrm{FAdV}-11)$. Mortality in infected flocks ranges between $5 \%$ and $10 \%$, but it can reach $30 \%$. The liver is the primary organ affected and is characterized by a pale, swollen, and friable appearance, in addition to petechial or ecchymotic hemorrhages (Hess 2013). The spleen can be enlarged and lesions can be seen on the kidneys. Histologically, the unique traits of infection with pathogenic FAdVs are the formation of inclusion bodies in the liver and multifocal vacuolar necrosis (Dar et al. 2012). FAdVs are transmitted both vertically and horizontally; the virus is excreted in high titres in the feces and transmission within a flock takes place via the fecal-oral route (Grgic et al. 2006; 2007). At present, there are no commercially available vaccines for IBH licensed for use in the United States and Canada. Given 
the economic impact of these viruses, availability of an effective vaccine is a priority, and correlating genetic changes with pathotype is critical for the development of vaccines and diagnostics.

Recently Zhao et al. (2015) published the sequences of two pathogenic FAdV-11 isolates, HBQ12 and BJH13 from China. In this study we sequenced and analyzed two FAdV-11 isolates from Ontario, Canada, one was obtained from a diseased flock diagnosed with IBH and the other one was isolated from an asymptomatic flock, allowing us to identify genetic markers responsible for the different pathotypes in FAdV-11 as well as comparison with the geographically distinct Chinese isolates.

Materials and methods

\section{Virus isolation and propagation}

Two Canadian FAdVs of Ontario origin were studied in detail. A pathogenic fowl aviadenovirus serotype 11 isolate was obtained from tissues collected from a flock showing clinical signs of $\mathrm{IBH}$, whereas the non-pathogenic serotype 11 isolate was recovered from a healthy flock; the viruses were designated as FAdV-11-ON-P2 and FAdV-11-ON-NP2, respectively. Virus isolation was done by Dr. Davor Ojkic (Animal Health Laboratories, University of Guelph, Ontario, Canada) (Ojkic et al. 2008a). The serotype of the isolates was established by sequence analysis of the hexon gene (Ojkic et al. 2008b). The viruses were plaque purified and propagated in permissive chicken hepatoma cells, the $\mathrm{CH}-\mathrm{SAH}$ cell line as previously described (Alexander et al. 1998). Plaque purification was performed by Dr. Helena Grgić (Grgić et al. 2014). CH-SAH cells were grown in Dulbecco's modified Eagle's medium/nutrient mixture F-12 Ham (DMEM-F12) supplemented with 10\% fetal bovine serum 
(FBS), $2 \mathrm{mM}$ L-glutamine, $100 \mathrm{U} / \mathrm{mL}$ penicillin, and $100 \mu \mathrm{g} / \mathrm{mL}$ streptomycin. For large scale virus production the cells were infected at a low multiplicity of infection (MOI). Cells and supernatant were collected when extensive cytopathic effect (CPE) was seen. Virus concentration and DNA extraction were performed as described (Ojkic and Nagy 2001). For the one step growth curves the $\mathrm{CH}-\mathrm{SAH}$ cells were infected with an MOI of 5 and samples were collected at specific hours post-infection (h.p.i.). The titers of the collected supernatants were determined by the plaque assay and expressed as plaque forming units per $\mathrm{mL}(\mathrm{PFU} / \mathrm{mL})$. The growth curves were repeated twice.

\section{Sequence analysis}

Viral DNA was sequenced using Illumina MiSeq next generation technology (Illumina, California, USA). Reads were compiled and formed into a single contig using DNA STAR SeqMan NGen12 primary sequence assembly (Applied Maths, Austin, TX). Regions of interest were amplified by PCR and sequenced to confirm identification of deletions, insertions, and repeats that were not present in FAdV-9.

\section{Bioinformatic analysis}

Predicted genes were calculated using Kodon (Applied maths, Austin, TX). For this study the criteria for determining open reading frames (ORFs) were as follows: ORFs must contain more than 75 nucleotides (nt), initiated with an ATG start codon, and must contain similarity to FAdV-9 (AC_000013, NC_000899.1) annotations in size, location, or splice sites. A previous study by Ojkic et al. (2002) was also taken into consideration. Potential splice sites were calculated using Alternate Splice Site Predictor (ASSP) program available online and crossreferenced to FAdV-9 (AC_000013) (Wang and Marín 2006). Sequences of predicted polypeptides of interest were aligned to translated sequences from known fowl adenovirus 
genomic information using CLUSTALW (Cost matrix of BLOSUM) (Larkin et al. 2007) to analyze structure and function. GC dinucleotides were calculated using the genomatix software suite (http://www.genomatix.de/cgi-bin/tools/tools.pl).

Phylogenetic trees were built and analyzed in Geneious $\mathrm{R}$ 8.0.3 (http;//www.geneious.com; Kearse et al. 2012) using RAxML 7.2.8 (protein model GAMMA LG, algorithm rapid bootstrapping and search for best-scoring ML tree replicated 1,000 times for statistical strength). Protein modeling was completed using Phyre 2 (Kelley et al. 2015). Binding analysis of protein was completed using 3DLigandSite-Ligand binding site prediction server on March $10^{\text {th }}, 2015$ (Wass et al. 2010).

\section{Nucleotide sequence accession numbers}

FAdV-11-ON-P2 and FAdV-11-ON-NP2 genomic information was submitted to GenBank under the accession numbers KU310942 and KP231537, respectively.

Nucleotide sequences utilized in this study can be accessed through GenBank: FAdV-11HBQ12 (KM096545), FAdV-11-BJH13 (KM096546), FAdV-9 (AC_000013, NC_000899.1), FAdV-1 (NC_001720), FAdV-8 (NC_014969), FAdV-4 (NC_015323), FAdV-5 (NC_021221), turkey adenovirus 1 (NC_014564).

Results

Two isolates of FAdV-11 that were isolated in Ontario poultry farms, one considered non-pathogenic (FAdV-11-ON-NP2) and one considered pathogenic (FAdV-11-ON-P2) were studied. One step growth curves of the two isolates in $\mathrm{CH}-\mathrm{SAH}$ cells were compared and are shown in Fig. 1. The FAdV-11-ON-P2 growth curve began to plateau at 30 h.p.i with a maximum titer at $2 \times 10^{8} \mathrm{PFU} / \mathrm{mL}$ which was reached by 48 h.p.i. In contrast the growth curve for 
the non-pathogenic isolate began to plateau earlier at 24 h.p.i. with a maximum titer of $2 \times 10^{7}$ PFU/mL, one log lower, reached at 48 h.p.i. The CPE induced by both viruses was similar to each other and typical for fowl aviadenoviruses. Moreover, there were no differences in plaque size and morphology.

The genomes of the two isolates were sequenced. Only reads with a Q score of 30 or greater were used, which resulted in 149 and 87 times coverage of FAdV-11-ON-P2 and FAdV11-ON-NP2, respectively. The length of the FAdV-11-ON-NP2 genome was 45,192 base pairs (bp) with a composition of $23.2 \% \mathrm{~A}, 27.5 \% \mathrm{C}, 26.5 \% \mathrm{G}, 22.8 \% \mathrm{~T}$, with a total $\mathrm{G}+\mathrm{C}$ content of 54\%. The FAdV-11-ON-P2 genome was 44,377 bp, with a composition of $23.3 \% \mathrm{~A}, 27.3 \% \mathrm{C}$, $26.4 \% \mathrm{G}, 23.0 \% \mathrm{~T}$, containing a total $\mathrm{G}+\mathrm{C}$ content of $53.7 \%$. Comparison of these FAdV -11 genomic sequences to other avian adenoviruses, including the recently published Chinese pathogenic FAdV-11 isolates (Zhao et al. 2015) is shown in Table 1. The lowest identity among the FAdV-11 isolates was between FAdV-11-ON-NP2 and the two Chinese FAdV-11 isolates, BJK13 (97.2.\%) and HBQ12 (97.5\%). The highest identity to the pathogenic ON isolate was with the two pathogenic Chinese isolates (98.8 to 98.9\%). Based on the criteria to predict ORFs a total of 37 coding regions on both the positive and negative strands were identified: 22 predicted ORFs located on the positive strand, and 15 predicted ORFs resided on the negative strand of both ON isolates. Homologs were identified by BLASTP using the non-redundant protein sequences database (Altschul et al. 1997), and the results for FAdV-11-ON-NP2 are shown in Table 2.

Phylogenetic analysis on both isolates was performed using Geneious Version 8.03. Specifically, the amino acid sequences of the $52 \mathrm{~K}$, hexon, and penton base were examined along with the nucleotide sequences for whole genomic comparison (Fig. 2). In each comparison, the 
two FAdV-11-ON isolates clustered most closely with each other and with the Chinese FAdV-11 isolates (Zhao et al. 2015) and were supported by high bootstrap values at all nodes. For the whole genome it was $100 \%$, $52 \mathrm{~K}$ was $99.1 \%$, hexon was $100 \%$ and penton base was $98.1 \%$. While the two Ontario strains could be resolved from the Chinese strains using the entire genome (Fig. 2A), they could not be based on the hexon, $52 \mathrm{~K}$ and penton bases amino acid sequences.

The predicted ORFs from FAdV-11 were analyzed using ClustalW. All four isolates contained identical hexon sequences, allowing any isolate to represent all FAdV-11 isolates in further analysis. The common hexon sequence from FAdV-11 was then compared to those of other fowl aviadenovirus species. The core regions showed a high degree of conservation, with several major regions showing hyper-variability at locations 140-220, 240-280, and 384-442 (Fig. 3). These three hyper-variable regions reside in the DE1 and FG1 regions of the hexon, known to be highly variable throughout human adenoviruses as well (Rux et al. 2003; Bruder et al. 2012).

In silico analysis revealed that the Ontario FAdV-11 isolates have one fiber gene, which is characteristic of FAdV-D virus. The fiber is responsible for viral attachment to host cells, facilitating binding between the penton base and integrin leading to receptor mediated endocytosis (Zhang and Bergelson 2005). The fiber of FAdV-11 has a well-conserved nuclear localization signal at positions 17-20 (RKRP), along with the VYPF motif associated with the interaction between the fiber and the penton base (Grgic et al. 2014). The isolates examined in this study contained only synonymous mutations in the fiber region, leading to no amino acid differences. While Grgic et al. (2014) examined the fiber sequence of multiple FAdV serotypes as a determining factor of pathogenicity, no correlation was found. 
The tandem repeat regions, TR-1 and TR-2, present in FAdV-9 were also identified in the Ontario FAdV-11 isolates. The TR-1 region of FAdV-11-ON-NP2 (nt 37,825 to nt 37,956) was found to be $33 \mathrm{bp}$ (one repeat) shorter than in FAdV-9, which was validated through independent PCR and sequence analysis. In addition, FAdV-11-ON-NP2 also contains a $135 \mathrm{bp}$ stretch that is repeated 13 times with a total length of 1755 bp (nt 38,933 to nt 40,688). This is identical to the TR-2 region of FAdV-9. In contrast, there is no TR-1 region in the pathogenic isolate FAdV-11ON-P2 and only 8 repeats of the 135 bp stretch were identified in the TR-2 region (nt 38,805 to nt 39,885). Zhao et al. (2015) sequenced two pathogenic FAdV-11 (HBQ12 and BJK13) isolates allowing for a comparison of the Canadian and Chinese viruses, which is summarized in Table 3. Similarly to FAdV-11-ON-P2, FAdV-11-BJK13 contained no TR-1 sequence, while FAdV-11HBQ12 contained 3 repeats. All 3 pathogenic isolates contained 8 or fewer repeats in the TR-2 region, whereas the non-pathogenic FAdV-11 and the non-pathogenic FAdV-9 have 13 TR-2 repeats. While the exact role of TR-2 remains to be determined, these findings suggest that the region may have a role in pathogenesis.

Genomic alignment of the two Ontario isolate showed 6 nonsynonymous mutations located in the reading frames encoding the IVa2 and DNA binding proteins.

The coding region for the $100 \mathrm{kDa}$ overlaps but is out of frame with $22 \mathrm{kDa}$, and $33 \mathrm{kDa}$ ORFs. The start site for the $33 \mathrm{~K}$ product is at $\mathrm{nt} 28,247$ and lies within the coding region for $100 \mathrm{~K}$ and is +1 out of frame allowing the production of transcripts with multiple ORFs. An additional 22K ORF which overlapped the $33 \mathrm{~K}$ ORF and started at the same nucleotide was present in only the two Ontario isolates. Two codon deletions were found in a glutamic acid rich region of the $22 \mathrm{~K}$ and $33 \mathrm{~K}$ ORFs, equivalent to the glycine rich region of $100 \mathrm{~K}$, of FAdV-11- 
ON-NP2. Three codons are deleted in the same region in FAdV-9, in the same species as FAdV11, affecting all three genes. No deletions were identified in the pathogenic viruses (Fig. 4). Adenovirus early gene products thwart the host's innate immune system and facilitate production of progeny viruses (Berk 2013). FAdV-11 also produces extensive transcripts early in infection. In this study we analyzed one gene product from the early transcriptional unit 6 (E6) located at the right end of the viral genome. E6 transcripts generate two predicted products from the same start codon, ORF23 and ORF23 alternative (ORF23a). ORF23 contains a transmembrane domain helix from amino acid position 258-278 (Kelley et al. 2015). A 3-D model of ORF23 was created with $95 \%$ of residues covered with $>90 \%$ confidence with Phyre 2 using multiple templates, which was then submitted to 3D Ligand Site to determine any putative binding motifs (Kelley et al. 2015). It was determined that ORF23 has a putative binding motif recognizing NAG (NAcetyl-D-Glucosamine, 1GYA), which is a glycosylation molecule found on an array of important surface receptors (Wyss et al. 1995). This binding domain was analyzed in depth and it is shown in Fig. 5. Late in infection ORF23 produces a variant, ORF23a through a splicing acceptor and donor recognized in the coding region, forming an $8 \mathrm{kDa}$ product (Ojkic et al. 2002). The donor site is found at 43,609 and the acceptor at position 43,029 , leading to a frame shift downstream of the splice acceptor resulting in an early stop codon at 42,932. Whether ORF23a is membrane bound or still recognizes NAG is not known and requires further study.

\section{Discussion}

Fowl aviadenoviruses are grouped into five species, and are comprised of both nonpathogenic and pathogenic variants, many of which are important to veterinary medicine and agriculture (Hess 2013). Due to the relatively low-cost and rapidity, next-generation sequencing 
is quickly becoming an additional resource for virus detection that has led to the recent identification of duck, turkey and pigeon adenoviruses (Marek et al. 2014a; 2014b). In this study we characterized and sequenced two FAdV-11 isolates taken from both diseased and asymptomatic chicken flocks in Ontario, Canada. The pathogenic isolate grew faster and to a higher titer in cell culture as seen in figure 1, representing increased replication efficiency. The genomes of the two FAdV-11 viruses, one non-pathogenic and one pathogenic, were assembled, annotated, and submitted to GenBank with accession numbers KP231537 and KU310942, respectively. Serotype 11 viruses are in Fowl aviadenovirus $D$ and our study increases the known FAdV-D genomic repertoire, allowing cross analysis among different isolates with varying phenotypes and pathotypes and different geographic origins. While Zhao et al. (2015) reported the sequences of two pathogenic Chinese FAdV-11 isolates, our comparative work allowed us to investigate potential genetic markers of virulence. Moreover, this study highlights the presence of identical serotypes, based on the identity of the hexon genes, present in Canada and China, and probably other parts of the world (Steer et al. 2015), which is key to understanding the epidemiology of the disease caused by this virus and allowing universal vaccine targeting against FAdV-11. Moreover the hexon protein is one of the primary antibody targets in vivo, encouraging epitope switching on key antigenic sites. Serotyping of FAdVs through sequencing and antigenic screening using the hexon gene is essential to understand the antibody mediated viral inhibition which is important in evaluating protective immunity.

Conserved mutations were found across the 5 genomes of viruses in FAdV-D in the coding region of the $100 \mathrm{~K}, 33 \mathrm{~K}$, and $22 \mathrm{~K}$ proteins. Both non-pathogenic isolates (FAdV-11-ONNP2 and FAdV-9) contained a single deletion composed of 2-3 amino acids, that affects three different ORFs (Fig. 4) with potential to alter protein structure and function. The $100 \mathrm{kDa}$ 
protein is responsible for the proper formation of the hexon trimer complex, mutations could influence the fluidity of the capsid, along with $33 \mathrm{kDa}$ and $22 \mathrm{kDa}$. The finding of this consistent mutation may be related to the molecular determinants of pathogenicity of adenoviruses. Similar mutations were noted for human adenoviruses, representing the wider implications of these findings in all adenoviruses. The three genes, which contain these potentially attenuating deletions, and their products are critical for viral replication. The $100 \mathrm{kDa}$ protein is involved in the formation of properly structured hexon trimers. Both, the $22 \mathrm{kDa}$ and $33 \mathrm{kDa}$ proteins are important splicing factors that allow complete transcripts to be expressed from the major late promoter (Morris et al. 2009). Interestingly, the $33 \mathrm{kDa}$ protein is also involved in packaging and it is critical in viral maturation, a $33 \mathrm{~K}$ deletion mutant produced empty viral particles (Wu et al. 2013). The $22 \mathrm{~K}$ was not identified in the Chinese isolates and in FAdV-9 possibly due to sequencing errors (Zhao et al. 2015; Ojkic and Nagy 2000)

The sizes of the genomes of the non-pathogenic FAdV-11 and FAdV-9, both in species D, are considerably larger than the pathogenic FAdV-11 (Zhao et al. 2015; Ojkic and Nagy 2000). The difference lies mainly in the TR-1 and TR-2 regions present at the right end of the genome. The non-pathogenic isolates contained higher numbers of copies of the repeats (Table 3.). The sequences of the repeats of FAdV-11 do not have any identity with the repeats of FAdV8 (Grgic et al. 2011). The function of these repeats is unknown, although we have shown for FAdV-9 that the TR-2 region is dispensable for viral replication in vitro and is a potential site for foreign gene insertion (Ojkic and Nagy 2001; 2003). Interestingly, tandemly repeated sequences have been reported in vaccinia and herpes viruses (Baroudy and Moss 1982; Umene et al. 1984). Understanding the sequence of the TR-2 itself has proven challenging, with a GC content of 
$\sim 75 \%$. We can only hypothesize about its interaction with the viral polymerase and local gene expression.

The adaptive immune system is critical in clearance of intracellular infections. It is in the virus's interest to inhibit this pathway to avoid the adaptive immune system. The putative functionality of ORF23 was examined in this study, highlighting its potential binding to NAG. One known immune related protein with NAG is the adhesion motif of human CD2, which is conserved across avian species (Vainio et al. 1991). Determining the binding partners of ORF23 requires future study, with implications in vaccine development.

In the Ojkic et al. studies it was found that ORF23 was alternatively spliced late in infection to produce an $8 \mathrm{kDa}$ protein ( $8 \mathrm{kDa}$ vs. $34.5 \mathrm{kDa}$ ) (Ojkic et al. 2002). The alternative splice product does not contain the original transmembrane domain, while maintaining the binding domain from amino acids at position 7-15. The role of the 31 amino acids encoded post the acceptor splice site is still under investigation. Determining whether ORF23a product is secreted or not is key in understanding the functionality of ORF23.

Other proteins that show potential in host immunomodulation are ORF25, ORF19, and ORF11. These proteins show putative domains responsible for lysosome interaction, phospholipase activity, and immunoglobulin like surface protein respectively.

This study identifies multiple candidate genetic markers for the pathogenesis of aviadenoviruses. Nevertheless, further studies are needed with pathogenic and non-pathogenic pairs of viruses of different serotypes to definitively identify genetic markers for virulence.

\section{Acknowledgements}


The authors thank Dr. Helena Grgić, Dr. Davor Ojkic, Mr. David Leishman and Mrs. BettyAnne McBey for their contribution and help. This work was supported by the Natural Sciences and Engineering Research Council of Canada (NSERC), the Canadian Poultry Research Council (CPRC), Agriculture and Agri-Food Canada and the Ontario Ministry of Agriculture, Food and Rural Affairs (OMAFRA).

\section{References}

Alexander, H.S., Huber, P., Cao, J., Krell, P.J., and Nagy, É. 1998. Growth characteristics of adenovirus type 8 in a chicken hepatoma cell line. J. Virol. Methods, 74: 9-14.

Altschul, S.F., Madden, T.L., Schaffer, A.A., Zhang, J., Zhang, Z., Miller, W., et al. 1997. Gapped BLAST and PSI-BLAST: a new generation of protein database search programs. Nucleic Acids Res. 25(1): 3389-3402.

Baroudy, B.M., and Moss, B. 1982. Sequence homologies of diverse length tandem repetitions near ends of vaccinia virus genome suggest unequal crossing over. Nucleic Acids Res. 10: 56735679.

Berk, A.J. 2013. Adenoviridae. In Fields Virology (6 $6^{\text {th }}$ edition). Edited by D.M. Knipe and P. Howley. Lippincott Williams and Wilkins, Philadelphia, P.A. Pp.1704-1731. 
Bruder, J.T., Semenova, E., Chen, P., Limbach, K., Patterson, N.B., Stefaniak, M.E., et al. 2012. Modification of Ad5 hexon hypervariable regions circumvents pre-existing Ad5 neutralizing antibodies and induces protective immune responses. PloS One 7(4): e33920.

Chiocca, S., Kurzbauer, R., Schaffner, G., Baker, A., Mautner, V., and Cotten, M. 1996. The complete DNA sequence and genomic organization of the avian adenovirus CELO. J. Virol. 70(5): 2939-2949.

Dar, A., Gomis, S., Shirley, I., Mutwiri, G., Brownlie, R., Potter, A., et al. 2012. Pathogenic and molecular characterization of a fowl adenovirus associated with inclusion body hepatitis in Saskatchewan chickens. Avian Dis. 56(1): 73-81.

Grgić, H., Philippe, C., Ojkić, D., and Nagy, É. 2006. Study of vertical transmission of fowl adenoviruses. Can. J. Vet. Res. 70: 230-233.

Grgić, H., Philippe, C., Ojkić, D., and Nagy, É. 2007. Serologic monitoring of a broiler breeder flock previously affected by inclusion body hepatitis and testing of the progeny for vertical transmission of fowl adenoviruses. Can. J. Vet. Res. 71: 98-102.

Grgic, H., Yang, D.H., and Nagy, É. 2011. Pathogenicity and complete genome sequence of a fowl adenovirus serotype 8 isolate. Virus Res. 156: 91-97. 
Grgic, H., Krell, P.J., and Nagy, É. 2014. Comparison of fiber gene sequences of inclusion body hepatitis (IBH) and non-IBH strains of serotype 8 and 11 fowl adenoviruses. Virus Genes, 48: 74-80.

Griffin, B.D., and Nagy, É. 2011. Coding potential and transcript analysis of fowl adenovirus 4: insight into upstream ORFs as common sequence features in adenoviral transcripts. J. Gen. Virol. 92: 1260-1272.

Hess, M. 2013. Aviadenovirus infections. In Diseases of poultry (13the edition). Edited by D.E., Swayne, J.R., Glisson, L.R., McDougald, L.K., Nolan, D.L. Suarez, and V., Nair. John Wiley \& Sons, Inc. Ames, IA. Pp. 289-300.

Kearse, M., Moir, R., Wilson, A., Stones-Havas, S., Cheung, M., Sturrock, S., et al. 2012.

Geneious Basic: an integrated and extendable desktop software platform for the organization and analysis of sequence data. Bioinformatics. 28(12): 1647-1649.

Kelley, L.A., Mezulis, S., Yates, C.M., Wass, M.N., and Sternberg, M.J. 2015. The Phyre2 web portal for protein modeling, prediction and analysis. Nat. Protoc. 10: 845-858.

Larkin, M.A., Blackshields, G., Brown, N.P., Chenna, R., McGettigan, P.A., and McWilliam, H. 2007. Clustal W and Clustal X version 2.0. Bioinformatics. 23(21): 2947-2948. 
Marek, A., Kosiol, C., Harrach, B., Kajan, G.L., Sclotterer, C., and Hess, M. 2013. The first whole genome sequence of a Fowl adenovirus B strain enables interspecies comparisons within the genus Aviadenovirus. Vet. Microbiol. 166(1-2): 250-256.

Marek, A., Ballmann, M.Z., Kosiol, C., Harrach, B., Schlotterer C., and Hess, M. 2014a. Wholegenome sequences of two turkey adenovirus types reveal the existence of two unknown lineages that merit the establishment of novel species within the genus Aviadenovirus. J. Gen. Virol. 95: $156-170$.

Marek, A., Kajan, G,L., Kosiol, C., Harrach, B., Schlotterer, C., and Hess, M. 2014b. Complete genome sequences of pigeon adenovirus 1 and duck adenovirus 2 extend the number of species within the genus Aviadenovirus. Virology, 462-463: 107-114.

Morris, S., and Leppard, K. 2009. Adenovirus serotype 5 L4-22K and L4-33K proteins have distinct functions in regulating gene expression. J. Virol. 83(7): 3049-3058.

Ojkic, D., and Nagy, É. 2000. The complete nucleotide sequence of fowl adenovirus type 8. J. Gen. Virol. 81: 1833-1837.

Ojkic, D., and Nagy, É. 2001. The long repeat region is dispensable for fowl adenovirus replication in vitro. Virology. 283: 197-206. 
Ojkic, D., and Nagy, É. 2003. Antibody response and virus tissue distribution in chickens inoculated with wild-type and recombinant fowl adenoviruses. Vaccine, 22: 42-48.

Ojkic, D., Krell, P.J., and Nagy, É. 2002. Unique features of fowl adenovirus 9 gene transcription. Virology, 302: 274-285.

Ojkic, D., Tuboly, T., Krell, P., and Nagy, É. 2008a. Characterization of fowl adenoviruses isolated in Ontario and Quebec, Canada. Can. J. Vet. Res. 72: 236-241.

Ojkic, D., Martin, E., Swinton, J., Vaillancourt, J., Boulianna, M., and Gomis, S. 2008b. Genotyping of Canadian isolates of fowl adenoviruses. Avian Pathol. 37(1): 95-100.

Rux, J.J., Kuser, P.R., and Burnett, R.M. 2003. Structural and phylogenetic analysis of adenovirus hexons by use of high-resolution x-ray crystallographic, molecular modeling, and sequence-based methods. J. Virol. 77: 9553-9566.

Steer, P.A., Sandy, J.R., O’Rourke, D., Scott, P.C., Browning, G.F., and Noormohammadi, A.M. 2015. Chronological analysis of gross and histological lesions induced by field strains of fowl adenovirus serotypes 1, 8b and 11 in one-day-old chickens. Avian Pathol. 44(2): 106-13.

Umene, K., Watson, R.J., and Enquist, L.W. 1984. Tandem repeated DNA in an intergenic region of Herpes simplex virus type 1 (Patton). Gene, 30: 33-39.

Vainio, O., Riwar, B., Brown, M.H., and Lassila, O. 1991. Characterization of the putative avian CD2 homologue. J. Immunol. 147(5): 1593-1599. 
Wang, M., and Marín, A. 2006. Characterization and prediction of alternative splice sites. Gene. 366: $219-227$.

Wass, M.N., Kelley, L.A., and Sternberg, M.J. 2010. 3DLigandSite: predicting ligand-binding sites using similar structures. Nucleic Acids Res. 38: 469-473.

Wu, K., Guimet, D., and Hearing, P. 2013. The adenovirus L4-33K protein regulates both late gene expression patterns and viral DNA packaging. J. Virol. 87(12): 6739-6747.

Wyss, D.F., Choi, J.S., Li, J., Knoppers, M.H., Willis, K.J., Arulanandam, A.R., et al. 1995. Conformation and function of the N-linked glycan in the adhesion domain of human CD2. Science, 269: 1273-1278.

Zhang, Y., and Bergelson, J.M. 2005. Adenovirus receptors. J. Virol. 79: 12125-12131.

Zhao, J., Zhong, Q., Zhao, Y., Hu, Y., and Zhang, G. 2015. Pathogenicity and complete genome characterization of fowl adenoviruses isolated from chickens associated with inclusion body hepatitis and hydropericardium syndrome in China. PloS one. DOI: 10.1371/journal.pone.0133073. 


\section{Figure captions}

Figure 1. Virus replication of FAdV-11 Ontario isolates in CH-SAH cells. The one step growth curves were established from the titers obtained for various times post-infection. The experiments were performed in duplicate, and the error bars represent the standard deviation.

Figure 2. Phylogenetic comparison of FAdV-11 genomes and genes. The phylogenetic trees were based on the nucleotide sequence of the full genome (panel A), and the amino acid sequences of the 52K (panel B), hexon (panel C), and penton base (panel D) genes, respectively. Sequences were aligned using ClustalW (BLOSUM cost matrix), and phylogenic analysis was in Geneious Version 8.03 using the RAxML 7.2.8 function (protein model GAMMA LG, algorithm rapid bootstrapping and search for best-scoring ML tree replicated 1,000 times for statistical strength). Bootstrap values are represented at each node.

Figure 3. Amino acid sequence analysis of the hypervariable region within the hexon protein of the aviadenovirus species. The alignments specifically analyze the DE1 (panel A) and FG1 (panel B) regions of the protein. The alignments of FAdV-11 (KP231537), FAdV-8 (NC_014969), FAdV-9 (AC_000013), FAdV-5 (NC_021221), FAdV-1 (NC_001720), and FAdV-4 (NC_015323) were performed using ClustalW. Stars (*) represent $100 \%$ similarity, periods (.) represent 67\%- 84\% similarity. Codon deletions for FAdV-11 ON-NP2 and FAdV-9 are shown as dashes in $\mathrm{A}$ and $\mathrm{B}$. Colour scheme used as defined at website http://ekhidna.biocenter.helsinki.fi/pfam2/clustal_colours.

Figure 4. Coding sequence overlap and alignment of three genes carrying a deletion (top panel). Amino acid analysis of codon deletions found in $100 \mathrm{~K}, 22 \mathrm{~K}$, and $33 \mathrm{~K}$ genes, which are present only in the non-pathogenic FAdV-D viruses (bottom panel). ClustalW was used for the analysis. The $22 \mathrm{~K}$ protein was not annotated in 3 of the 5 studied viruses.

Figure 5. Ligand binding site of the predicted product of ORF23. The 3D model of ORF23 was generated using Phyre 2, with NAG (N-Acetyl-D-Glucosamine, 1GYA) added using 3DLigandSite. Amino acids coloured blue are responsible for ligand interaction. The green atoms present represent the metallic ion present in the ligand. A table on the right shows the corresponding amino acids responsible for the binding domain of NAG. Residue refers to the location of the amino acid within the protein. Contact is the number of ligands from similar matrixes, in which that specific amino acid is within 0.8 angstrom. Average distance is the average distance that the molecule is from the amino acid, 0.00 means they are directly touching. 
Table 1. Comparison of FAdV-11 genomes to other serotypes of fowl adenoviruses.

\begin{tabular}{|c|c|c|c|c|c|c|c|}
\hline \multirow[t]{2}{*}{ Serotype } & \multirow{2}{*}{$\begin{array}{l}\text { Genome } \\
\text { size (bp) }\end{array}$} & \multirow{2}{*}{$\begin{array}{l}\text { Percent GC } \\
(\%)\end{array}$} & \multirow[t]{2}{*}{ CG dinucleotides } & \multicolumn{4}{|c|}{ Identity (\%) } \\
\hline & & & & FAdV-11-ON-NP2 & FAdV-11-ON-P2 & FAdV-11-HBQ12 & FAdV-11-BJK13 \\
\hline $\begin{array}{l}\text { FAdV-11-ON-NP2 } \\
\text { (KP231537) }\end{array}$ & 45,192 & 54.1 & 3,214 & - & 98.1 & 97.5 & 97.2 \\
\hline $\begin{array}{l}\text { FAdV-11-ON-P2 } \\
\text { (KU310942) }\end{array}$ & 44,377 & 53.7 & 3,107 & 98.1 & - & 98.8 & 98.9 \\
\hline $\begin{array}{l}\text { FAdV-11-HBQ12 } \\
\text { (KM096545) }\end{array}$ & 44,080 & 53.6 & 3,054 & 97.5 & 98.8 & - & 99.7 \\
\hline $\begin{array}{l}\text { FAdV-11-BJK13 } \\
\text { (KM096546) }\end{array}$ & 43,961 & 53.5 & 3,051 & 97.2 & 98.9 & 99.7 & - \\
\hline $\begin{array}{l}\text { FAdV-9 } \\
\text { (AC_000013) }\end{array}$ & 45,063 & 53.8 & 3,178 & 95.7 & 93.9 & 93.3 & 93.0 \\
\hline $\begin{array}{l}\text { FAdV-8 } \\
\text { (NC_014969) }\end{array}$ & 44,055 & 57.9 & 3,587 & 68.0 & 67.9 & 68.2 & 68 \\
\hline $\begin{array}{l}\text { FAdV-5 } \\
\text { (NC_015323) }\end{array}$ & 45,781 & 56.5 & 3,563 & 60.5 & 60.6 & 60.7 & 60.5 \\
\hline $\begin{array}{l}\text { FAdV-4 } \\
\text { (NC_015323) }\end{array}$ & 45,667 & 54.6 & 3,287 & 53.3 & 54.1 & 54.3 & 54.2 \\
\hline $\begin{array}{l}\text { FAdV-1 } \\
\text { (NC_001720) }\end{array}$ & 43,804 & 54.3 & 3,109 & 53.6 & 54.0 & 54.0 & 54.0 \\
\hline
\end{tabular}


Table 2. Predicted gene products of FAdV-11-ON-NP2. Predicted open reading frames were analyzed using BLASTP to determine homology and function.

\begin{tabular}{|c|c|c|c|c|c|c|}
\hline Gene & Location (bp) & Strand & Length (aa) & Description & $\begin{array}{l}\text { Closest viral homolog } \\
\text { (GenBank accession no.) }\end{array}$ & Identity (\%) \\
\hline ORFO & $575-809$ & + & 77 & Undetermined & FAdV-2 (DQ208708.1) & 100 \\
\hline ORF1 & $847-1,339$ & + & 163 & dUTPase & FAdV-9 (NC_000899.1) & 100 \\
\hline ORF1A & $1,339-1,498$ & + & 52 & Undetermined & FAdV-2 (DQ208708.1) & 100 \\
\hline ORF1B & $1,500-1,731$ & + & 76 & Undetermined & FAdV-2 (DQ208708.1) & 100 \\
\hline ORF1C & $1,735-1,879$ & + & 47 & Undetermined & FAdV-2 (DQ208708.1) & 98 \\
\hline ORF2 & $1,952-2,756$ & + & 267 & Undetermined & FAdV-2 (DQ208708.1) & 100 \\
\hline ORF24 & $16,288-2,838$ & - & 228 & Undetermined & FAdV-9 (AC_000013.1) & 99 \\
\hline ORF14 & $16,288-3,537$ & - & 230 & Undetermined & FAdV-9 (AC_000013.1) & 100 \\
\hline ORF13 & $16,288-4,263$ & - & 330 & Undetermined & FAdV-9 (AC_000013.1) & 100 \\
\hline ORF12 & $16,288-5,245$ & - & 307 & Undetermined & FAdV-9 (AC_000013.1) & 99 \\
\hline IVa2 & $7,337-6,131$ & - & 401 & IVa2 multifunctional protein & FAdV-9 (NC_000899.1) & 99 \\
\hline DNA pol & $11,254-7,333$ & - & 1306 & Viral DNA polymerase & FAdV-9 (NC_000899.1) & 99 \\
\hline pTP & $16,288-11,250$ & - & 648 & Precursor terminal protein & FAdV-9 (AC_000013.1) & 99 \\
\hline $52 \mathrm{~K}$ & $13,247-14,456$ & + & 402 & Late encapsidation protein & FAdV-9 (NC_000899.1) & 97 \\
\hline pllla & $14,442-16,218$ & + & 591 & pllla protein precursor & FAdV-9 (AC_000013.1) & 97 \\
\hline III & $16,298-17,936$ & + & 545 & Penton base protein & FAdV-2 (KT862805.1) & 100 \\
\hline pVII & $17,976-18,213$ & + & 78 & Core protein & FAdV-9 (AC_000013.1) & 92 \\
\hline $\mathrm{pX}$ & $18,450-19,050$ & + & 199 & Mu peptide precursor & FAdV-9 (NC_000899.1) & 100 \\
\hline $\mathrm{pVI}$ & $19,177-19,864$ & + & 228 & Minor capsid protein precursor & FAdV-9 (NC_000899.1) & 100 \\
\hline hexon & $19,976-22,829$ & + & 950 & capsid protein & FAdV-8 (NC_014969.1) & 90 \\
\hline protease & $22,842-23,460$ & + & 205 & Protease & FAdV-9 (NC_000899.1) & 99 \\
\hline DBP & $25,345-23,575$ & - & 550 & DNA binding protein & FAdV-9 (AC_000013.1) & 96 \\
\hline $100 \mathrm{~K}$ & $25,409-28,574$ & + & 1055 & $100 \mathrm{kDa}$ hexon assembly protein & FAdV-9 (NC_000899.1) & 94 \\
\hline $22 \mathrm{~K}$ & $28,251-28,800$ & + & 182 & 22 kDa encapsidation protein & FAdV-9 (AC_000013.1) & 97 \\
\hline $33 \mathrm{~K}$ & $28,251-29,163$ & + & 222 & $33 \mathrm{kDa}$ protein & FAdV-9 (AC_000013.1) & 97 \\
\hline pVIII & $29,202-29,928$ & + & 241 & Hexon associated protein & FAdV-9 (NC_000899.1) & 99 \\
\hline U exon & $30,180-29,946$ & - & 78 & U exon & $\begin{array}{l}\text { FAdV-9 (AC_000013.1) and } \\
\text { FAdV-8 (AF021254.1) }\end{array}$ & 100 \\
\hline fiber & $30,178-31,897$ & + & 572 & Fiber protein & FAdV-9 (NC_000899.1) & 85 \\
\hline ORF22 & $32,529-31,956$ & - & 190 & Undetermined & FAdV-2 (EF458160.1) & 99 \\
\hline
\end{tabular}


Table 2. Predicted gene products of FAdV-11-ON-NP2. Predicted open reading frames were analyzed using BLASTP to determine homology and function.

$\begin{array}{lllllll}\text { ORF20A } & 34,044-32,533 & - & 166 & \text { Undetermined } & \text { FAdV-9 (AC_000013.1) } & 94 \\ \text { ORF20 } & 34,044-33,012 & - & 312 & \text { Undetermined } & \text { FAdV-9 (AC_000013.1) } & 97 \\ \text { ORF19 } & 36,468-34,245 & - & 714 & \text { Triacylglycerol lipase } & \text { FAdV-2 (EF458160.1) } & 99 \\ \text { Gam-1 } & 37,993-38,827 & + & 277 & \text { Putative sumo interacting protein } & \text { FAdV-2 (EF458160.1) } & 100 \\ \text { ORF17 } & 41,202-40,731 & - & 156 & \text { Undetermined } & \text { FAdV-2 (EF458160.1) } & 100 \\ \text { ORF11 } & 41,596-42,530 & + & 260 & \text { Undetermined } & \text { FAdV-9 (AC_000013.1) } & 99 \\ \text { ORF23 } & 43,726-42,790 & - & 311 & \text { Putative NAG interacting protein } & \text { FAdV-9 (NC_000899.1) } & 97 \\ \text { ORF25 } & 44,178-44,767+ & 169 & \text { Undetermined } & \text { FAdV-9 (AC_000013.1) } & 99\end{array}$


Table 3. Genomic comparison of repeats in isolates of Fowl aviadenovirus D.

\begin{tabular}{llll}
\hline Isolate & $\begin{array}{l}\text { TR-1 } \\
\text { \# of repeats }\end{array}$ & $\begin{array}{l}\text { TR-2 } \\
\text { \# of repeats }\end{array}$ & $\begin{array}{l}\text { Contains } \\
\text { mutation of } \\
\text { interest* }^{*}\end{array}$ \\
\hline FAdV-11-BJK13 & 0 & 7 & No \\
FAdV-11-HBQ12 & 3 & 7 & No \\
FAdV-11-ON-P2 & 0 & 8 & No \\
FAdV-11-ON-NP2 & 4 & 13 & Yes \\
FAdV-9 & 5 & 13 & Yes \\
\hline
\end{tabular}

TR-1: tandem repeat region 1; TR-2: tandem repeat region 2

* The mutation of interest is the two-three codons deleted in $100 \mathrm{~K}, 22 \mathrm{~K}$ and $33 \mathrm{~K}$ regions 


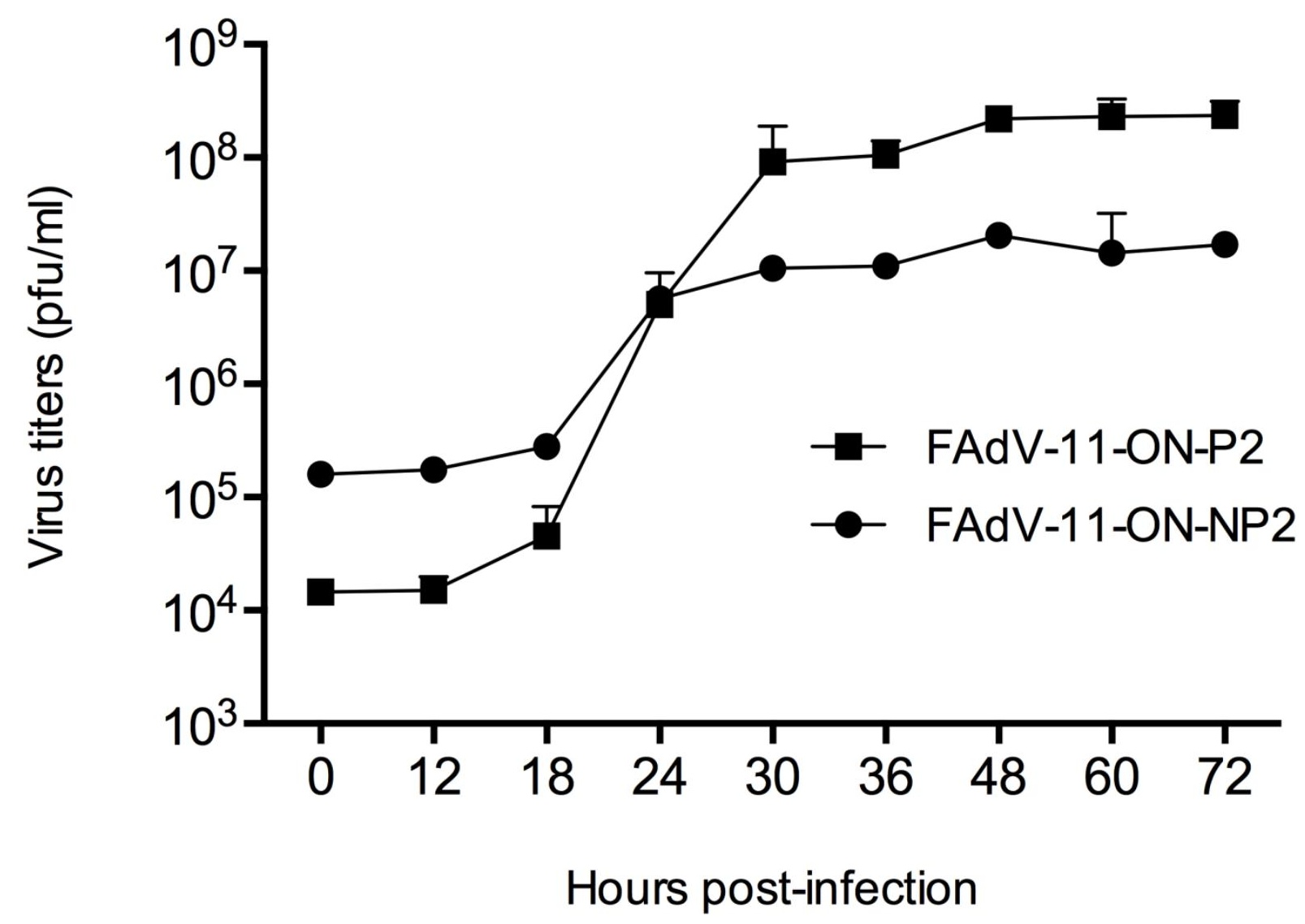



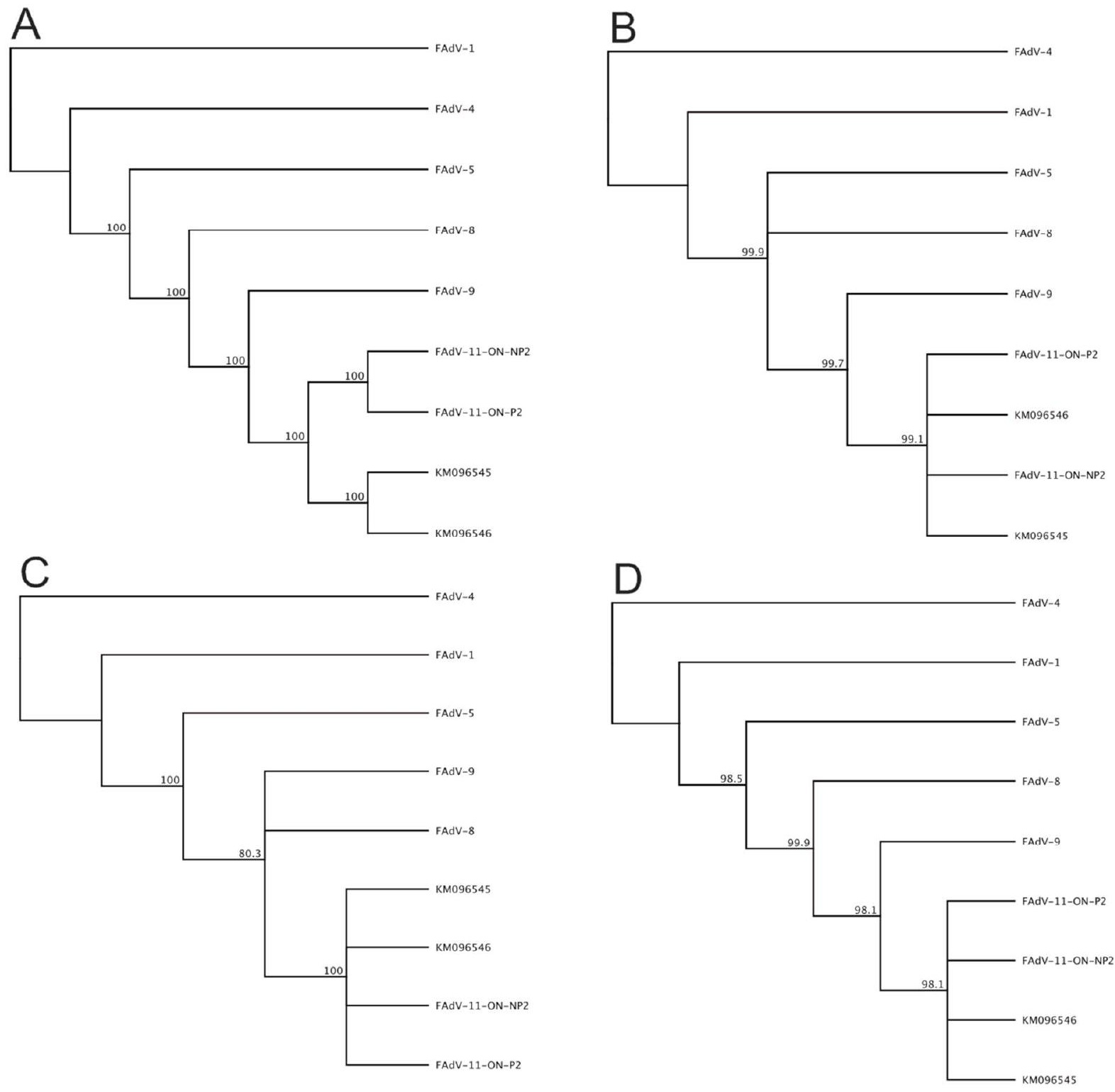

120
130
140
150
160
170

FAdV - 11

PYGGTAYNPLÄPREAFFNNWVDTEASK- - TVITGQMTTPYEN-VQGAKDKTAAIV FAdV - 8

FAdV - 9

FAdV - 5

FAdV - 1

FAdV - 4

FAdV - 11

FAdV - 8

FAdV - 9

FAdV - 5

FAdV - 1

FAdV - 4

PYGGTAYN P LAPRE AF FNNWIEDDGNN- - TT T GQMTNPYKNEAQNTATATAAA I

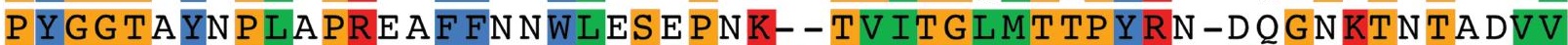
P YGGTAYN P LAPREALFNNMLVST SNTPQTVITGQMTHPYANETNTDPTKTAAAI P Y GG T A YN P LA PRE A I FN TWVEST G P - - TNVVGQMTNVYTNQT RN DKT A T LQV P YCGTAYN P LAPKE S MFN NWSETAP GQ- NVS AS GQL SNVYTN - T S T T KD T T A AQV

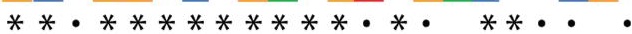

180

190

210

220

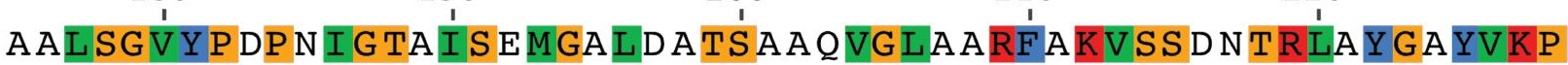
A S V SG S Y P NP N V G LA I S E VGA L T P TLA A QVG LA G RF A K V SN E N T R LA Y G A YVK P

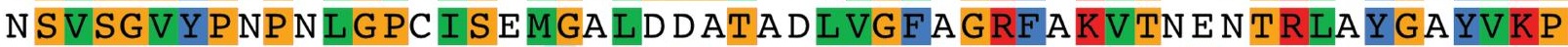
A S MSG TVPNPN L G PC I E MPA LA TAS A E N VG L MARF A I GA T N K LAYGAYVKP N S I SG VVP NVN L G PG L S Q LASR - - ADVDN IGVVGRF A KVDS A GVKQAYG AYVKP TKI SG VF P N N Q G PG IN - - - P L RQVENANTGVLGRFAKS QYN - - - YAYGAYVKP

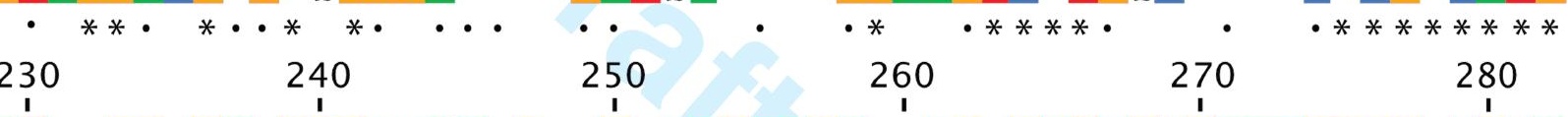

FAdV - 11

FAdV - 8

FAdV - 9

FAdV - 5

FAdV - 1

FAdV - 4

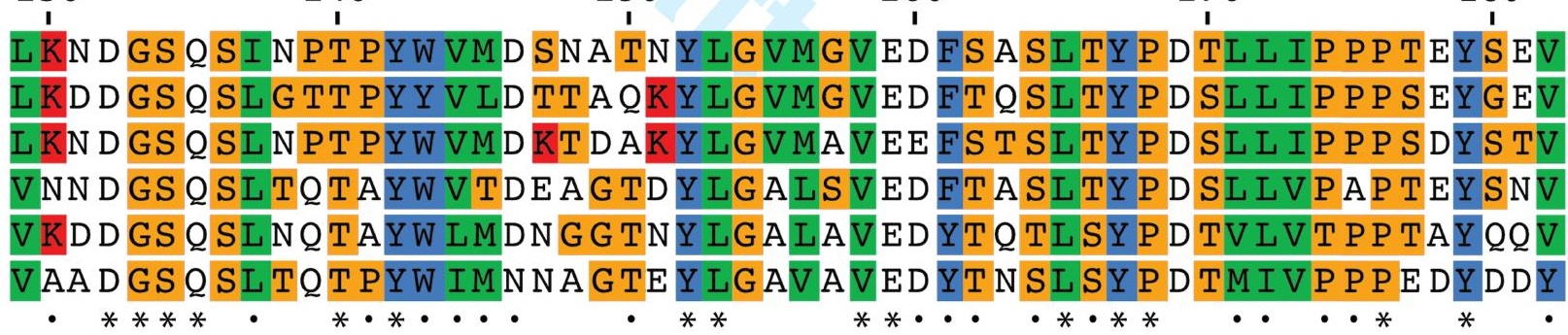

$390 \quad 400 \quad 410 \quad 420 \quad 430$

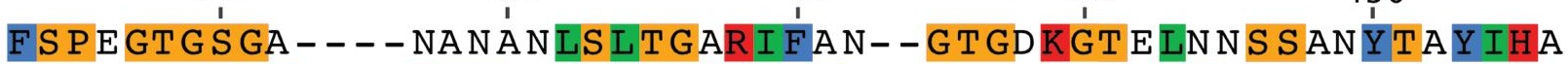
F S P E GT GQGP - - - - IS S AN ITLS GVKVYTN- - GQNDKGT EVTN - - - L T TY LNA F S P E GT GYGA - - - NPN SN LS FA DVKVHQN- - GA GDKGNE INT G GATYS TY LTV F S P E GT G S GS G LA TAD A T T F S F A QVKVWEN- - G S GA K GN ELTN - - - Y G TY LHA F L P D GHGA GE - - - - - DNG PDL SNVK I Y TN- - GQQDKG VVVA - - -TVS TQLNF

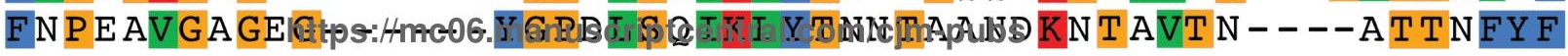
$* * \cdot \cdots * *$

FAdV - 11 FAdV - 8 FAdV - 9 FAdV - 5 FAdV - 1 FAdV - 4 
Start codon for $33 \mathrm{~K}$ and $22 \mathrm{~K}$
located at nucleotide
position: $28,289-91$

28,300

28,350

28,400

28,450

28,500

Stop codon for $100 \mathrm{~K}$

Located at nucleotide

position: $28,615-7$

\section{FAdV-11-HBQ12}

FAdV-11-BJ K13

FAdV-11-ON-P2

FAdV-11-ON-NP2

FAdV-9

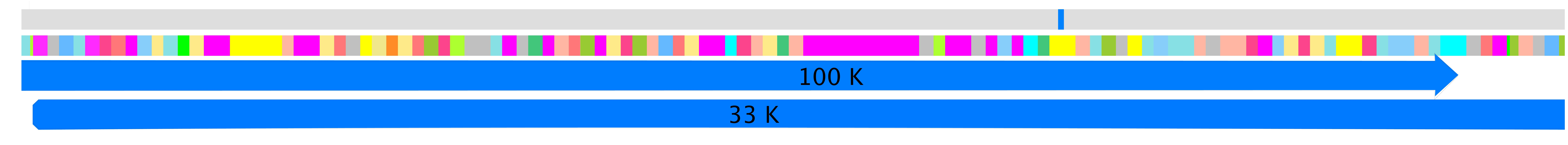

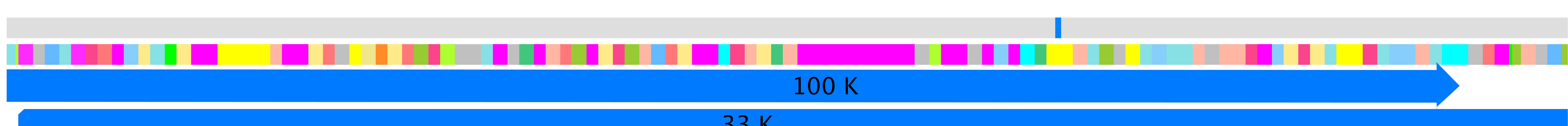

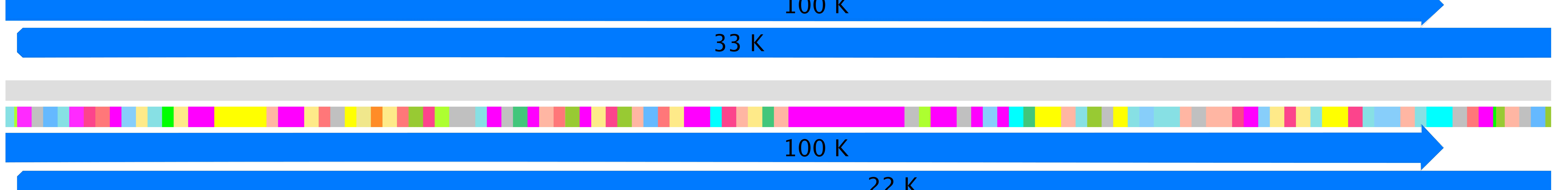
$33 \mathrm{~K} \quad 22 \mathrm{~K}$

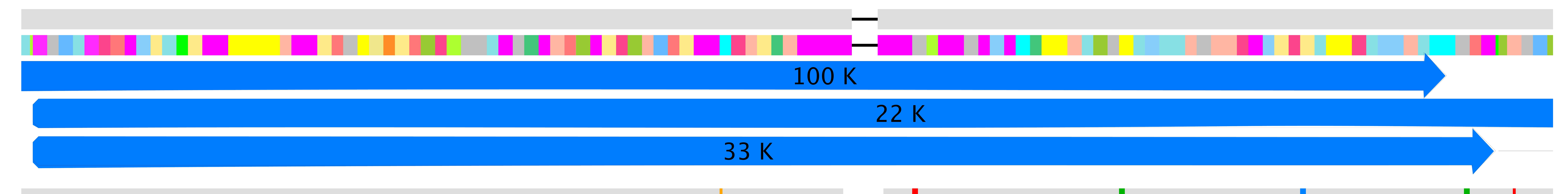
\begin{tabular}{|l|l|l|l|l|}
\hline \\
\hline
\end{tabular}

Splice site donor for $33 \mathrm{~K}$ at nucleotide

B

\section{K}

position: 28,629

FAdV-11-HBQ12 FAdV-11-BJ K13 FAdV-11-ON-P2 FAdV-11-ON-NP2 FAdV-9

50

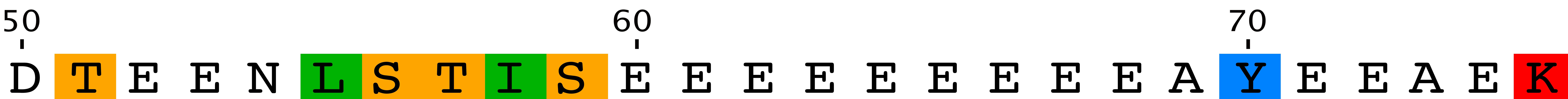
D T E E N L S T I S E E E E E E E E E A Y E E A E K D T E E N L S T I S E E E E E E E E E A Y E E A E K D T E E N L S T I E E E E E E E - A Y E E E K $\mathrm{D} T \mathrm{TE}$ L $\mathrm{S} T \mathrm{~T}$ S E E E E E T - - Y E E A E K

\section{$22 \mathrm{~K}$}

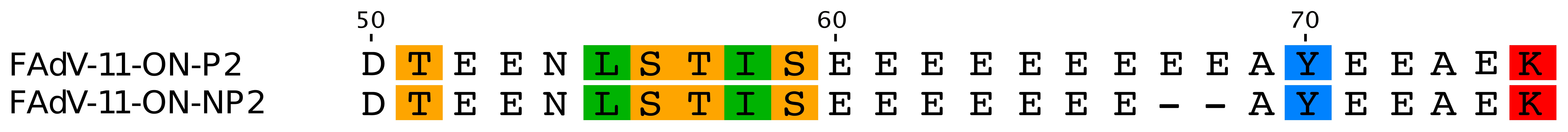

\section{$100 \mathrm{~K}$}

FAdV-11-HBQ12

FAdV-11-BJ K13

FAdV-11-ON-P2

FAdV-11-ON-NP2

FAdV-9

1,000

1,010

1,020

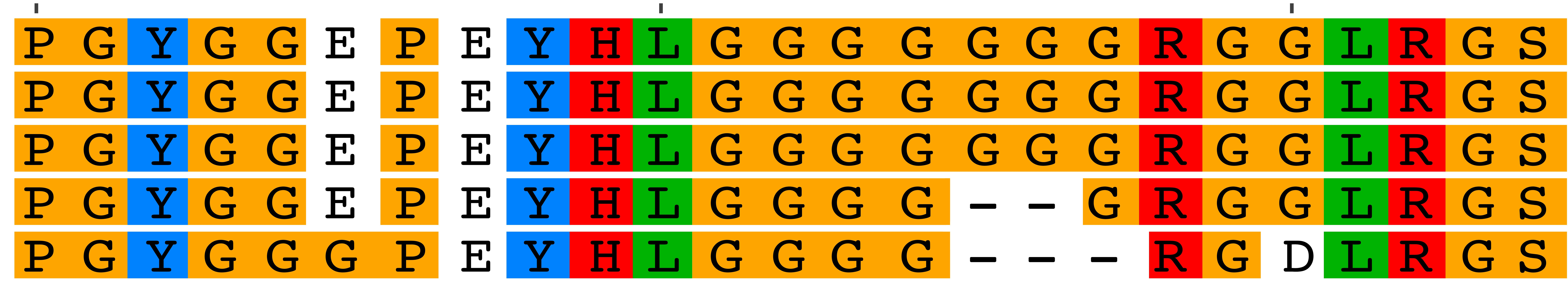




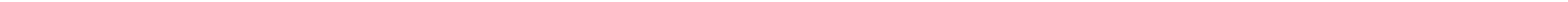

